\title{
Incorporating a disturbance observer with direct velocity feedback for control of human-induced vibrations
}

\author{
Dr Donald Nyawako, Professor Paul Reynolds, Dr Emma Hudson \\ Vibration Engineering Section, College of Engineering, Mathematics and Physical Sciences, University of \\ Exeter, EX4 4QF, U.K.e-mail: d.s.nyawako@exeter.ac.uk, p.reynolds@exeter.ac.uk, e.j.hudson@exeter.ac.uk
}

\begin{abstract}
Feedback control strategies are desirable for disturbance rejection of human-induced vibrations in civil engineering structures as human walking forces cannot easily be measured. In relation to human-induced vibration control studies, most past researches have focused on floors and footbridges and the widely used linear controller implemented in the trials has been the direct velocity feedback (DVF) scheme. With appropriate compensation to enhance its robustness, it has been shown to be effective at damping out the problematic modes of vibration of the structures in which the active vibration control systems have been implemented.

The work presented here introduces a disturbance observer (DOB) that is used with an outer-loop DVF controller. Results of analytical studies presented in this work based on the dynamic properties of a walkway bridge structure demonstrate the potential of this approach for enhancing the vibration mitigation performance offered by a purely DVF controller. For example, estimates of controlled frequency response functions indicate improved attenuation of vibration around the dominant frequency of the walkway bridge structure as well as at higher resonant frequencies. Controlled responses from three synthesized walking excitation forces on a walkway bridge structure model show that the inclusion of the disturbance observer with an outer loop DVF has potential to improve on the vibration mitigation performance by about $3.5 \%$ at resonance and 6-10\% off-resonance. These are realised with hard constraints being imposed on the low frequency actuator displacements.
\end{abstract}

Keywords: Direct velocity feedback (DVF), disturbance observer (DOB), vibration control, human-induced vibrations

\section{INTRODUCTION}

Integrated control systems can enhance controller designs through meeting fundamental objectives such as reference tracking, disturbance rejection, stabilization and robustness. These series of goals can be achieved with a combined approach as opposed to the use of just a single feedback controller system ${ }^{1}$. Additional considerations in the form of vibration mitigation performances, potential installation and running costs, robustness and efficiency of such systems have to be taken into account in any potential project proposal.

In relation to civil structures subjected to human-induced vibrations, for example floors and footbridges, feedback control strategies have been trialled in field studies to augment damping of structural resonant frequencies prone to such excitations. This has been instrumental towards improving their vibration serviceability performances. This approach has been adopted because human walking forces cannot easily be measured. The controller scheme used in most of such past studies is direct velocity feedback (DVF) due to its robustness ${ }^{2,3,4}$.

Feedback control systems can suffer from some inherent disadvantages, for example their inability to provide some form of predictive control to compensate for the effects of measurable or unmeasurable disturbances which may in turn present unsatisfactory vibration mitigation performances. An example is the inability of predominantly feedback systems to deal with off-resonance problems, for example, which may lie in the quasistatic zone ${ }^{5}$. To deal with some of these problems, approaches incorporating disturbance observers have been explored to compensate for these limitations. Previous research has demonstrated disturbance estimators combined with feedback controller schemes such as the Independent Modal Space Control (IMSC) to suppress vibrations in both linear and nonlinear flexible structures such as a beam and flexible boom ${ }^{5,6}$. This has had an impact in improving the vibration mitigation performance beyond only a feedback system with an IMSC controller. It has been acknowledged that one of the key challenges in implementing disturbance-compensated feedforward control is dependent on the accuracy of the structural model used. 
In instances where a purely feedback control system does not present satisfactory vibration mitigation performance, some additional improvements in the control can be realised by the addition of some form of feedforward control. There has been a vast amount of research dedicated to the parameterization of disturbance observers as well as an overview of their equivalence in linear systems ${ }^{7,8,9}$, their design for compensation of low frequency disturbances ${ }^{10}$, as well as implementations in the control of a high-speed direct drive positioning table and in position control of lightweight single-link flexible arms with large payload variations ${ }^{11,12}$.

The motivation for the work presented here is to investigate a potential approach for dealing with both onresonance and off-resonance excitations of civil engineering structures from human activities. Human activities contain energy not only at the activity frequency but also at harmonics of this frequency. Where the harmonics coincide with resonant frequencies of a structure, this has potential for exciting resonance. On the other hand, where this does not coincide with exciting resonance, the amount of energy may still be sufficiently significant to cause annoyance or disturbance. Previous controllers, for example, the direct velocity feedback (DVF) controller have impressive vibration attenuation properties at resonance, but are inefficient at dealing with the effects of disturbances that may be even slightly off-resonance. Addition of a controller loop that enhances the vibration mitigation performance of such a controller off-resonance would be beneficial. In this paper, sections 2 and 3 introduce the actuator and walkway bridge dynamics, and section 4 introduces the design of controller schemes: direct velocity feedback (DVF) without and with a disturbance observer (DOB). Section 5 presents the results of analytical studies in terms of the actuator displacement to disturbance input estimates, estimates of frequency response functions (FRF) and uncontrolled and controlled responses to synthesized walking forces.

\section{ACTUATOR}

The actuator whose dynamics are used in the analytical studies presented in this work is an APS Dynamics electrodynamic shaker, model 400 . The analytical models representing the force-voltage (N/V) and displacementvoltage $(\mathrm{m} / \mathrm{V})$ characteristics when the actuator is driven in the current drive mode can be represented by Equations 1 and 2. The actuator parameters are estimated as: $K_{a c t}=300 \mathrm{~N} / \mathrm{V}, \zeta_{\text {act }}=0.10, \omega_{\text {act }}=8.17 \mathrm{rad} / \mathrm{s}$, and $K_{a c t \_d}=10$. The actuator stroke is $\pm 75 \mathrm{~mm}$. A notch filter in Equation 3, with $k_{\text {not }}=8$ is introduced to compensate for the low damping characteristic when the actuator is driven in the current drive mode which has the potential to introduce a stroke saturation instability. Figures $1 \mathrm{a}$ and $1 \mathrm{~b}$ show the frequency response function (FRF) magnitude and phase of the force-voltage characteristics for both the uncompensated and compensated actuator dynamics.

$$
\begin{aligned}
& G_{a c t}(s)=\frac{F(s)}{V(s)}=\frac{K_{a c t} s^{2}}{s^{2}+2 \zeta_{a c t} \omega_{a c t} s+\omega_{a c t}^{2}} \\
& G_{a c t \_d}(s)=\frac{D(s)}{V(s)}=\frac{K_{a c t \_d}}{s^{2}+2 \zeta_{a c t} \omega_{a c t} s+\omega_{a c t}^{2}} \\
& G_{n o t}(s)=\frac{s^{2}+2 \zeta_{a c t} \omega_{a c t} s+\omega_{a c t}^{2}}{s^{2}+2 k_{n o t} \zeta_{a c t} \omega_{a c t} s+\omega_{a c t}^{2}}
\end{aligned}
$$

Where $\quad \begin{array}{ll}G_{a c t}(s) & \text { Actuator dynamics (Force - Voltage) } \\ G_{a c t \_d}(s) & \text { Actuator dynamics (Displacement - Voltage) } \\ F(s) & \text { Actuator force } \\ V(s) & \text { Voltage input } \\ K_{a c t} & \text { Actuator force - voltage characteristic } \\ K_{a c t_{-} d} & \text { Derived actuator displacement - voltage characteristic } \\ \zeta_{a c t} & \text { Damping ratio of actuator } \\ \omega_{a c t} & \text { Resonant frequency of actuator } \\ k_{\text {not }} & \text { Notch filter constant }\end{array}$




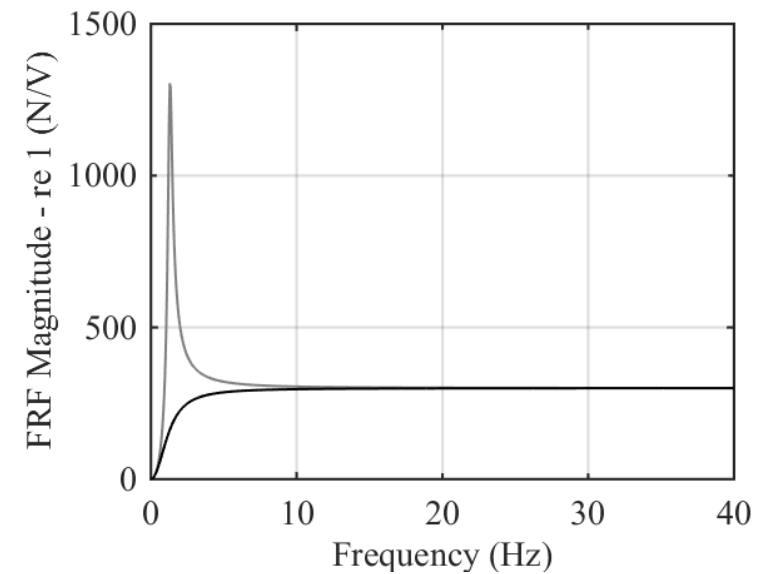

a)

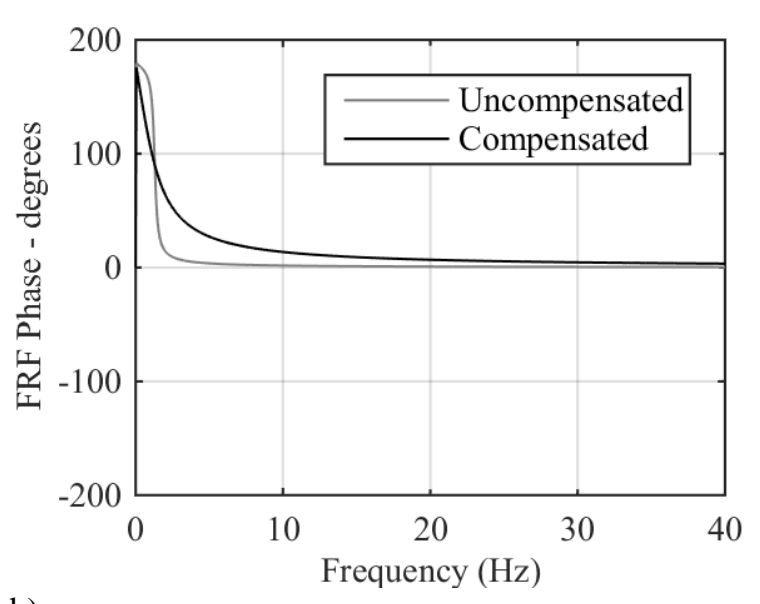

b)

ristics) with and without notch filter compensation

\section{WALKWAY BRIDGE DYNAMICS}

The walkway bridge located in the University of Exeter's Forum building is the structure whose dynamic properties are used in the analytical work presented here. This is shown in Figure $2 \mathrm{a}$ and Figure $2 \mathrm{~b}$ shows a plan view of the test points that were used for experimental modal analysis (EMA) tests. Further details of these tests are given in $^{13}$. A summary of the estimated modal properties of the lowest four vibration modes observable at Test Point (TP) 7 are presented in Table 1. TP7 is the sensor/actuator location for the control studies in this work.

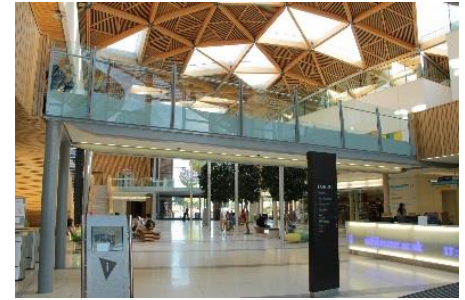

a)

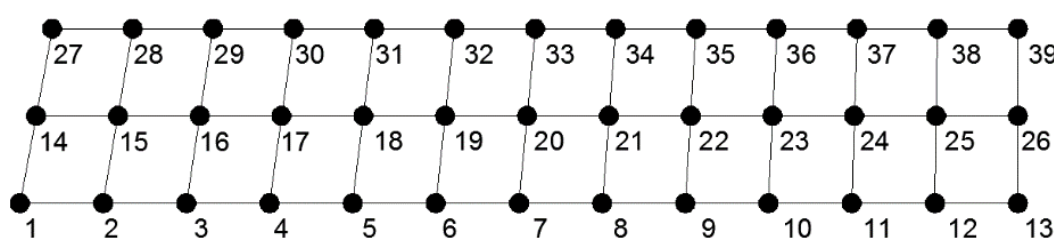

b)

Figure 2. (a) Photo of walkway bridge in the Forum building, University of Exeter and (b) a plan view of the test grid used for EMA

Table 1. Summary of estimated modal properties from EMA

\begin{tabular}{|c|c|c|}
\hline Mode & Natural Frequency [Hz] & Damping ratio [\%] \\
\hline 1 & 6.33 & 1.0 \\
\hline 2 & 10.5 & 0.9 \\
\hline 3 & 14.6 & 2.0 \\
\hline 4 & 34.3 & 2.6 \\
\hline
\end{tabular}

From the estimated modal properties, a lumped parameter model of the walkway bridge with ' $n$ ' modal coordinates can be derived as shown in Equation 4. $M^{*}, C^{*}$ and $K^{*}$ are the $n \times n$ modal mass, modal damping and modal stiffness matrices. $D$ is the $m \times m$ actuator location matrix and $E$ is the $m \times m$ excitation force location matrix. $\emptyset$ represents the $m \times n$ mass normalised modal transformation matrix and $\zeta_{i}$ and $\omega_{i}$ are the modal damping ratio and circular natural frequency of the $i$ th vibration mode. State space representations in Equations 5 and 6 can be obtained from Equation 4, and they express the output in the form of modal displacements and velocities. The transformation $\emptyset^{T} x_{1}$ and $\emptyset^{T} x_{2}$ can be used to revert back to the nodal displacements and velocities in the physical space at all sensor locations in the EMA tests. Thus, reduced order models (ROMs) at different points of interest in the walkway bridge structure, necessary for controller designs as well as for analytical studies can be developed as shown in Equation 7. These can be converted to transfer function form through the transformation $G(s)=C_{p o}\left(s I-A_{p o}\right)^{-1} B_{p o}+D_{p o} . \mathrm{TP} 7$ in Figure $2 \mathrm{~b}$ is the location selected for the control studies in this work. 


$$
\begin{aligned}
& M^{*} \ddot{x}+C^{*} \dot{x}+K^{*} x=\emptyset^{T} D u+\emptyset^{T} E f \\
& \left\{\begin{array}{l}
\dot{x}_{1} \\
\dot{x}_{2}
\end{array}\right\}=\left[\begin{array}{cc}
0 & I \\
-\frac{K^{*}}{M^{*}} & -\frac{C^{*}}{M^{*}}
\end{array}\right]\left\{\begin{array}{l}
x_{1} \\
x_{2}
\end{array}\right\}+\left[\begin{array}{cc}
0 & 0 \\
\emptyset^{T} D & \emptyset^{T} E
\end{array}\right]\left\{\begin{array}{l}
u \\
f
\end{array}\right\} \\
& \left\{\begin{array}{l}
y_{1} \\
y_{2}
\end{array}\right\}=\left[\begin{array}{ll}
I & 0 \\
0 & I
\end{array}\right]\left\{\begin{array}{l}
x_{1} \\
x_{2}
\end{array}\right\}+\left[\begin{array}{ll}
0 & 0 \\
0 & 0
\end{array}\right]\left\{\begin{array}{l}
u \\
f
\end{array}\right\} \\
& \dot{x}=A_{p o} x+B_{p o} u, \quad y=C_{p o} x+D_{p o} u
\end{aligned}
$$

Where

$$
\begin{array}{rlrl}
K^{*} & =\left[\begin{array}{cccc}
\omega_{1}^{2} & 0 & \cdots & 0 \\
0 & \omega_{2}^{2} & \cdots & 0 \\
\vdots & \vdots & \ddots & \vdots \\
0 & 0 & \cdots & \omega_{n}^{2}
\end{array}\right] & C^{*}=\left[\begin{array}{cccc}
2 \zeta_{1} \omega_{1} & 0 & \cdots & 0 \\
0 & 2 \zeta_{2} \omega_{2} & \cdots & 0 \\
\vdots & \vdots & \ddots & \vdots \\
0 & 0 & \cdots & 2 \zeta_{n} \omega_{n}
\end{array}\right] \\
M^{*}=\left[\begin{array}{cccc}
1 & 0 & \cdots & 0 \\
0 & 1 & \cdots & 0 \\
\vdots & \vdots & \ddots & \vdots \\
0 & 0 & \cdots & 1
\end{array}\right] & \emptyset=\left[\begin{array}{cccc}
\emptyset_{1,1} & \emptyset_{1,2} & \cdots & \emptyset_{1, m} \\
\emptyset_{2,1} & \emptyset_{2,2} & \cdots & \emptyset_{2, m} \\
\vdots & \vdots & \ddots & \vdots \\
\emptyset_{n, 1} & \emptyset_{39,2} & \cdots & \emptyset_{n, m}
\end{array}\right]
\end{array}
$$

\section{DIRECT VELOCITY FEEDBACK WITHOUT AND WITH DISTURBANCE OBSERVER}

The controller scheme for the DVF controller designed and implemented in the analytical studies is shown in Figure 3. $G_{p}(s), G_{a c t}(s), G_{b p}(s), G_{n o t}(s)$ and $C(s)$, in which $s=j \omega$, represent the walkway bridge, actuator, band pass filter, notch filter and controller dynamics. $d_{i}(t), \ddot{y}_{p}(t), f_{c}(t), r(t), e(t)$, and $v(t)$ are the disturbance inputs, structural acceleration responses, actuator force, reference signal, error signal and control signal.

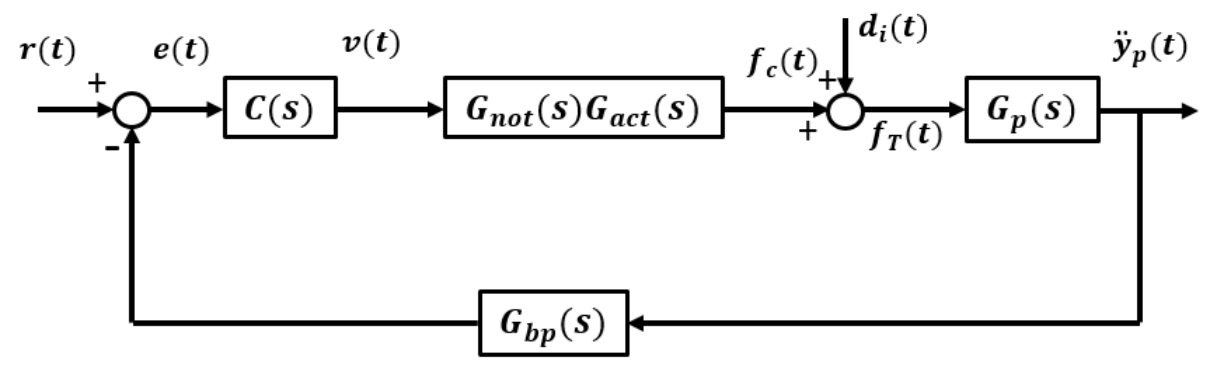

Figure 3. DVF controller

For the mitigation of human-induced vibrations, the augmentation of structural damping through feedback as shown in Equation 9 has often been the primary objective of the control design in previous studies as human walking forces cannot directly be measured. The DVF controller, $C(s)$, takes the form of Equation 8 , and $K_{g}$ is the gain parameter to be designed. The design of $K_{g}$ is often a compromise between performance and stability requirements. Typical requirements set out to be met by the DVF controller to ensure its robustness are:

1. To meet minimum stability margins, i.e. Gain Margin (GM) of 5dB and Phase Margin (PM) of 30 degrees.

2. The peak of the actuator displacement to disturbance input relationship in Equation 10 around the actuator resonant frequency, i.e. $s=j \omega_{\text {act }}$ should not exceed the threshold of $0.05 \mathrm{~mm} / \mathrm{N}$. This is a dynamic quantity that minimises potential for stroke saturation from the harmonics of walking around the actuator resonant frequency.

$$
C(s)=\frac{K_{g}}{s}
$$




$$
\begin{aligned}
& y_{p}(s)=\frac{G_{p}(s)}{1+G_{p}(s) G_{n o t}(s) G_{a c t}(s) G_{c}(s) G_{b p}(s)} d_{i}(s) \\
& y_{a c t_{-} d}(s)=-\frac{G_{n o t}(s) G_{a c t \_d}(s) G_{c}(s) G_{b p}(s) G_{p}(s)}{1+G_{n o t}(s) G_{a c t \_d}(s) G_{c}(s) G_{b p}(s) G_{p}(s)} d_{i}(s)
\end{aligned}
$$

For the DVF controller, a gain of $K_{g}=1150 \mathrm{Vs} / \mathrm{m}$ satisfies the stability margins (GM and PM) outlined by requirement (1) above, whilst a gain of $K_{g}=800 \mathrm{Vs} / \mathrm{m}$ satisfies the requirement (2) above. Thus, the minimum of the two gains i.e. $K_{g}=800 \mathrm{Vs} / \mathrm{m}$ is the driver for the maximum gain that can be implemented with the purely DVF controller. $G_{b p}(s)$ is a second order Butterworth bandpass filter with cut-off frequencies $0.5-50.0 \mathrm{~Hz}$.

An outer loop DVF controller with a disturbance observer in a frequency domain formulation is shown in Figure $4^{8}$. The disturbance observer is a compensatory controller that makes use of the output of the disturbance observer to impose disturbance cancellation as an additional feature to the outer loop DVF controller. This is investigated in this work to deal with off-resonant disturbances, which are quite common from the harmonics of human loading patterns on civil engineering structures. The outer loop DVF controller still augments the open-loop structural damping. The additional parameters in Figure 4, i.e. $Q(s), G_{n}^{-1}(s)$ and $G$ represent a second order Butterworth band pass filter, inverse plant dynamics and a gain term to convert the estimated disturbance force into a command voltage signal.

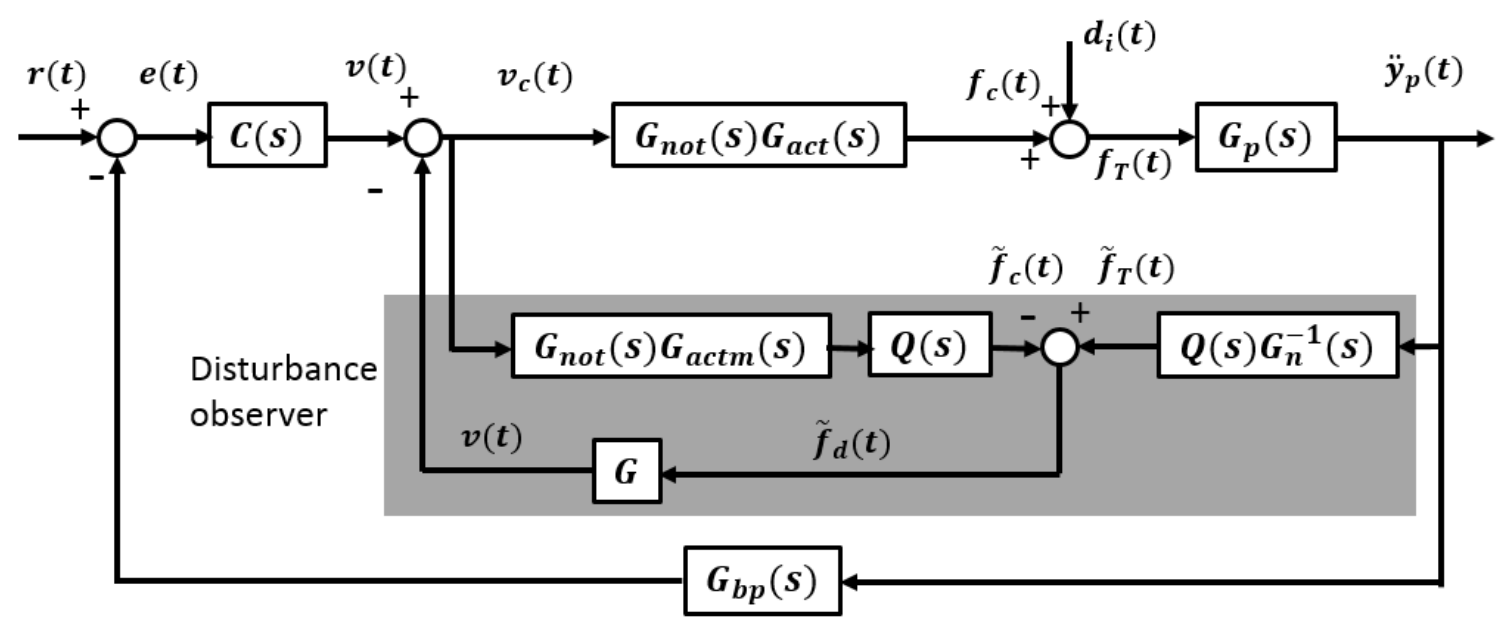

Figure 4. Outer loop DVF controller with disturbance observer (DOB)

$C(s)$ is the same form as in Equation 8, and the relationships in Equations 11 to 14 can also be developed for the DVF controller with a disturbance observer. $G_{n}^{-1}$ is the inverse plant dynamics based on the fundamental vibration mode of the walkway bridge considered in $G_{p}(s)$ from Table 1. $Q(s)$ is a second order band pass Butterworth filter with cut-off frequency of $3.5-20.0 \mathrm{~Hz}$ and the gain term $G=0.00125$ in this work. These are the key design parameters for the disturbance observer loop.

$$
\begin{aligned}
& y_{p}(s)=\frac{G_{p}(s)}{1+G_{p}(s) G_{n o t}(s) G_{a c t}(s) G_{c}(s)\left(C(s)-G_{Q}(s)\right)} d_{i}(s) \\
& y_{\text {act_d }}(s)=-\frac{G_{n o t}(s) G_{a c t_{-} d}(s) G_{c}(s) G_{b p}(s) C(s) G_{p}(s)}{1+G_{n o t}(s) G_{a c t_{-} d}(s) G_{c}(s) G_{b p}(s) C(s) G_{p}(s)} d_{i}(s) \\
& G_{Q}(s)=Q(s) G_{n}^{-1}(s) G \\
& G_{c}(s)=\frac{1}{1+Q(s) G_{n o t}(s) G_{a c t m}(s) G}
\end{aligned}
$$




\section{RESULTS OF ANALYTICAL STUDIES}

The analytical studies presented comprise of:

1. A review of the actuator displacement to disturbance input relationships in Equations 10 and 12 for the DVF and DVF+DOB controllers.

2. Estimates of uncontrolled and controlled frequency response functions (FRFs) for DVF and DVF+DOB controllers.

3. Uncontrolled and controlled responses to synthesized walking excitation forces at three different pacing frequencies for DVF and DVF+DOB controllers as well as monitoring of the actuator displacements.

\subsection{Actuator displacements under DVF and DVF+DOB}

The predictions of the actuator displacement to disturbance input relationships for the DVF and DVF+DOB controllers in Equations 10 and 12 for the velocity gain designed: $K_{g}=800 \mathrm{Vs} / \mathrm{m}$ are shown in Figure 5. They represent implementations with the notch filter compensation in Equation 3. Both DVF and DVF+DOB meet the required design constraint of not exceeding the design limit imposed of $0.05 \mathrm{~mm} / \mathrm{N}$. This is shown for the frequency bandwidth $0-5 \mathrm{~Hz}$ which is more critical in the evaluation of the potential for actuator stroke saturation. At higher frequencies, actuator stroke does not present a problem and hence this is not shown here.

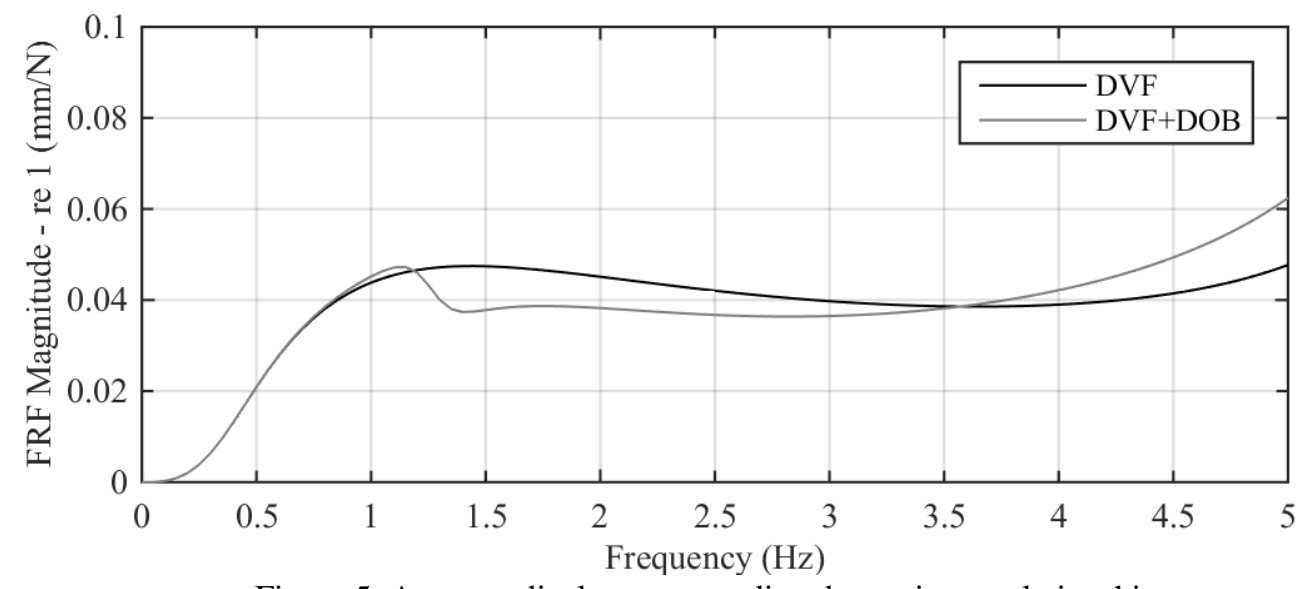

Figure 5. Actuator displacement to disturbance input relationships

\subsection{Uncontrolled and controlled frequency response functions (FRFs)}

The estimates of the uncontrolled and controlled FRFs are given in Figures $6 a$ and $6 \mathrm{~b}$, in which the latter is a zoomed plot of the former that is introduced for clarity. OL and CL refer to open-loop and closed-loop systems, respectively. CL DVF1 and CL DVF+DOB1 represent Equations 9 and 11 without notch filter compensation for the actuator dynamics whilst CL DVF and CL DVF+DOB represent the same equations with notch filter compensation for the actuator dynamics. Estimates of controlled FRFs for CL DVF1 and CL DVF+DOB1 highlight the influence of the notch filter used to provide compensation for the actuator dynamics in the slight degradation of performance for DVF and DVF+DOB by virtue of the phase it introduces. The estimates of the controlled FRFs in Figure $6 \mathrm{~b}$ show that that the DVF+DOB controller has better vibration mitigation performances at and around the dominant resonant frequency of the walkway bridge structure as well as at and around the higher frequencies of the walkway bridge structure in comparison to the DVF controller. 


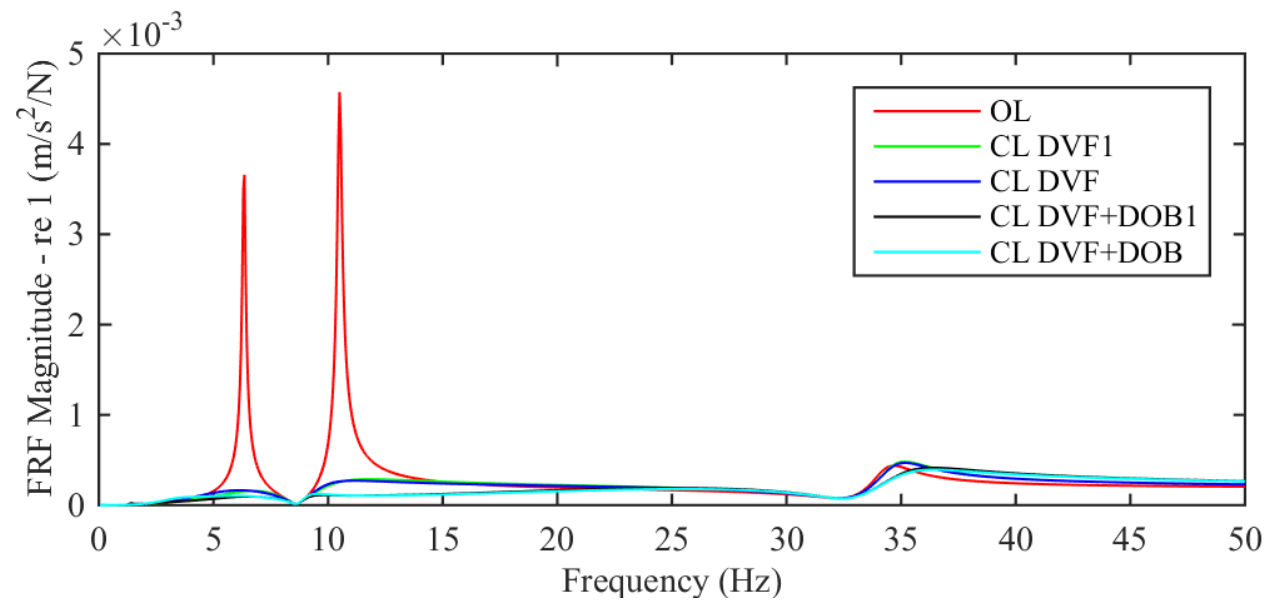

a)

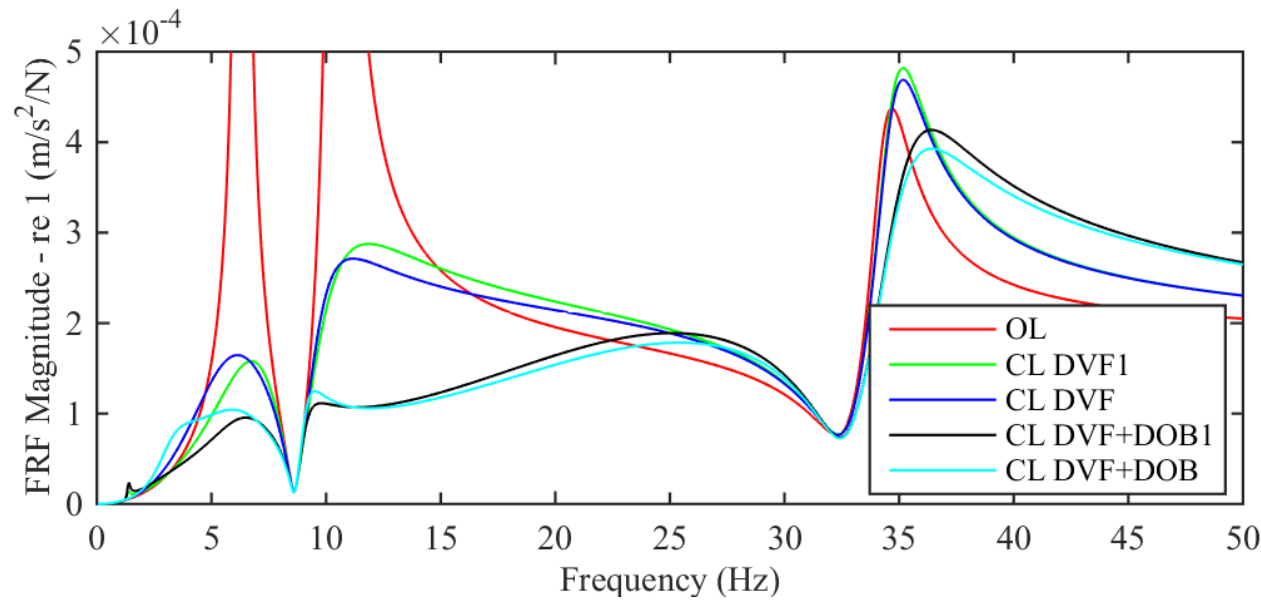

b)

Figure 6. DVF and DVF+DOB controllers with and without inner loop actuator compensation: a) over the entire frequency bandwidth considered, b) a zoomed plot around controlled resonances

\subsection{Responses to walking excitation}

Three synthesized walking excitation forces, described here as walking frequencies 1, 2 and 3, were generated and introduced as disturbance inputs, $d_{i}(t)$ in the systems described by Figures 3 and 4 . They represent realistic input forces that are derived from an actual acceleration response time history measured at TP7 for a pedestrian walking across the walkway bridge structure at a pacing frequency of $2.1 \mathrm{~Hz}$, which is the third sub-harmonic of its fundamental resonant frequency. This is fed through the inverse plant dynamics and appropriate filtering applied to generate the synthesized walking force in Figure 7 a for walking frequency 1 . The sampling time steps were adjusted to generate walking frequencies 2 and 3 in Figures $7 \mathrm{~b}$ and 7c. These correspond with walking frequencies of $1.9 \mathrm{~Hz}$ and $1.7 \mathrm{~Hz}$. The associated Fourier spectra of the three walking frequencies 1,2 and 3 in Figure 7 are shown in Figure 8. 


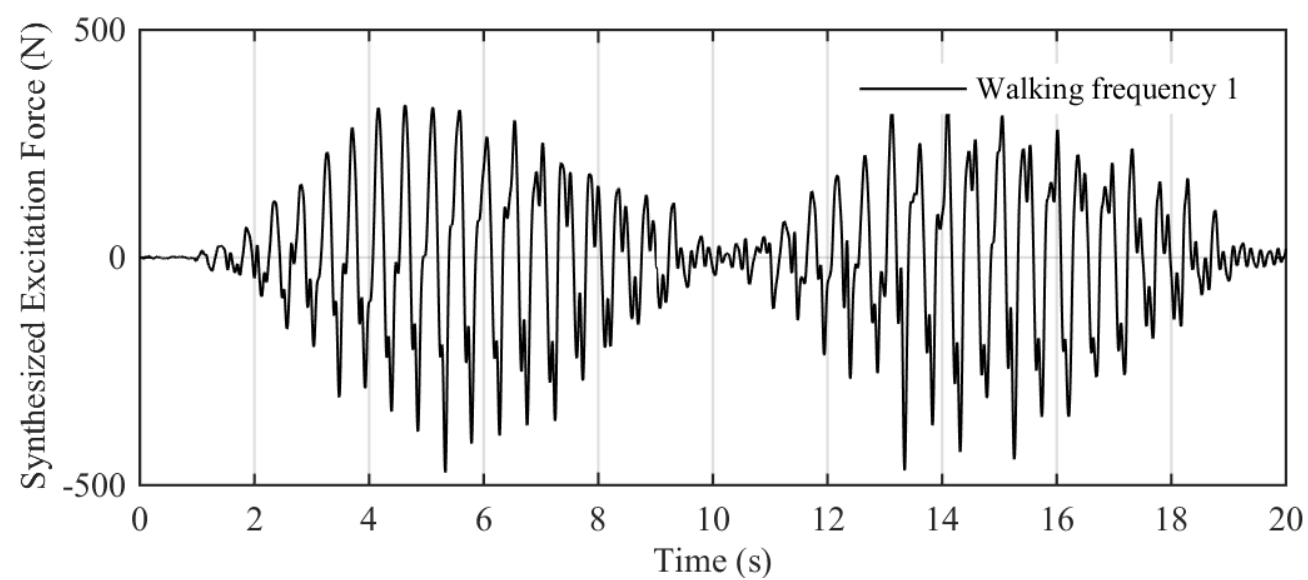

a) Walking at $2.1 \mathrm{~Hz}$

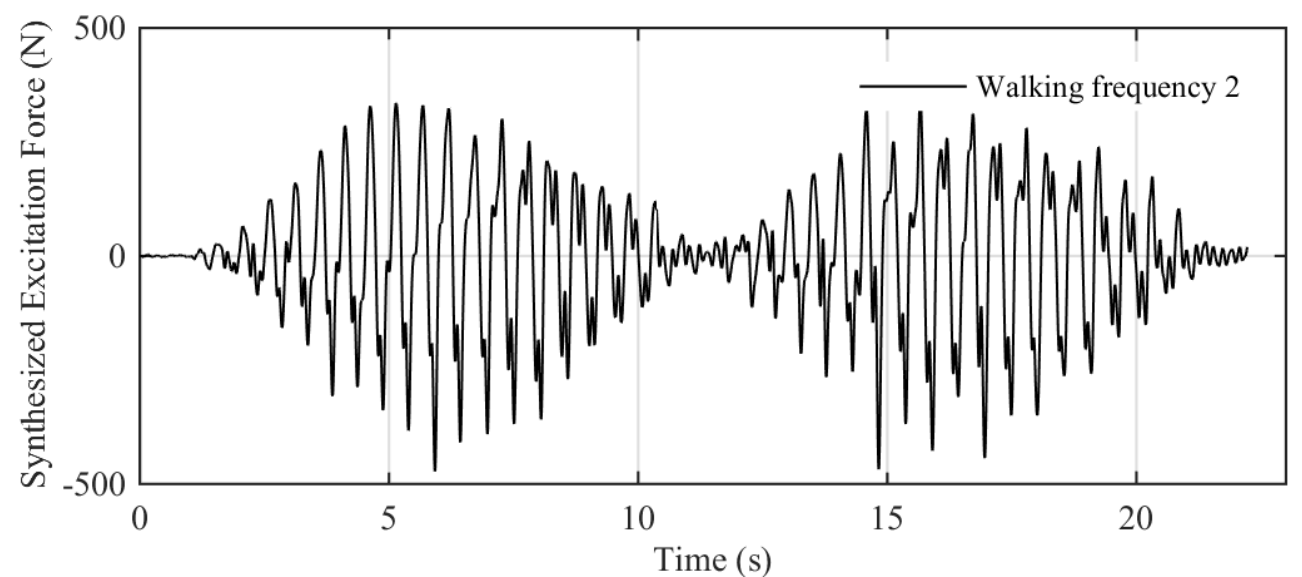

b) Walking at $1.9 \mathrm{~Hz}$

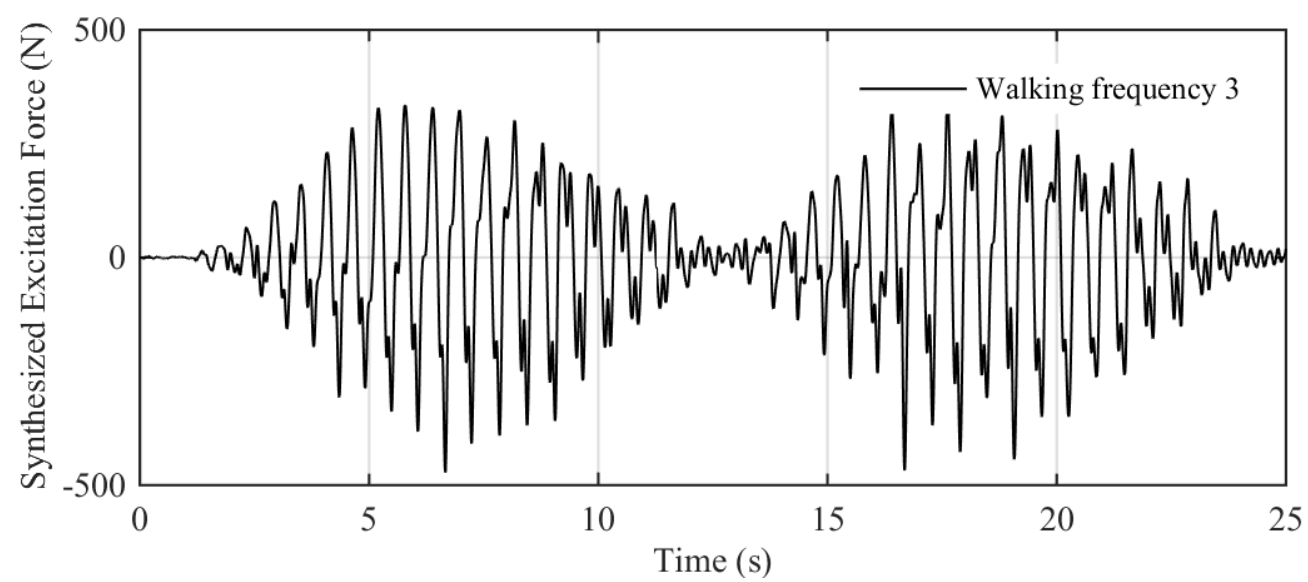

c) Walking at $1.7 \mathrm{~Hz}$

Figure 7. Synthesized walking force time histories at three different walking frequencies of $2.1 \mathrm{~Hz}, 1.90 \mathrm{~Hz}$ and $1.7 \mathrm{~Hz}$ 


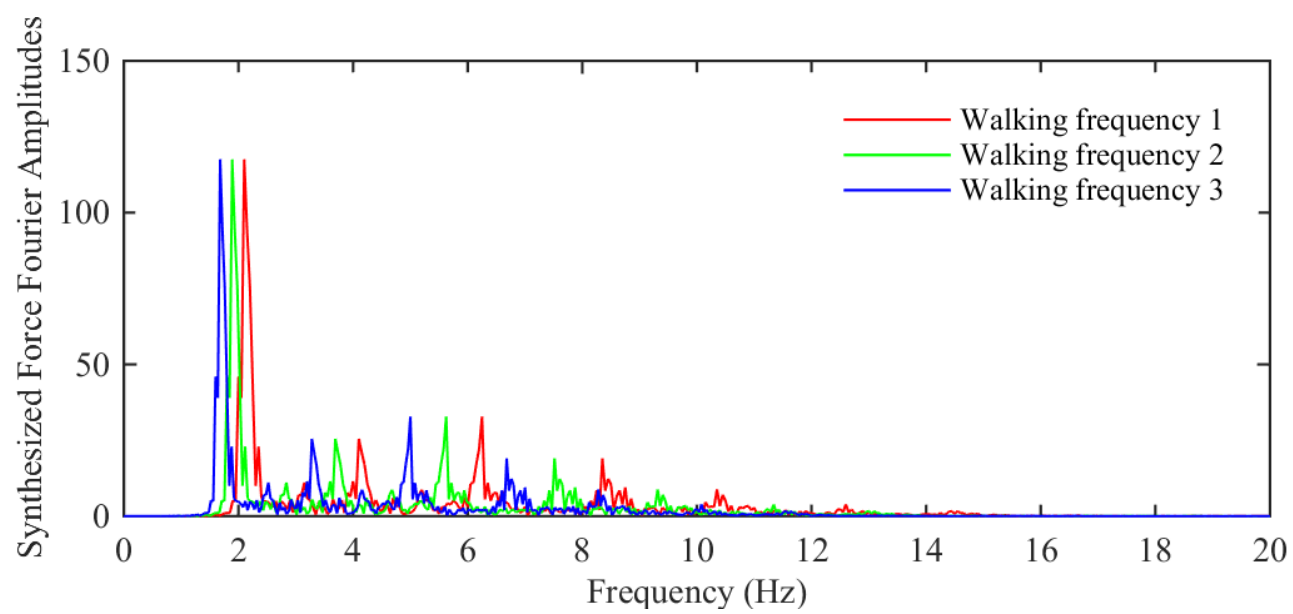

Figure 8. Fourier spectra of synthesized walking force time histories in Figure 7

Figures 9, 10 and 11 show the estimates of the uncontrolled and controlled structural acceleration responses as well as the actuator forces. Table 2 gives an indication of the improvement in performance for both the DVF and DVF+DOB controllers, these being implemented with compensation for the actuator dynamics. These are the peaks of the 1s running root mean square (RMS) acceleration responses, which are defined as the maximum transient vibration values (MTVV) following the recommendation of the International Organization of Standardization: ISO $2631^{14}$.

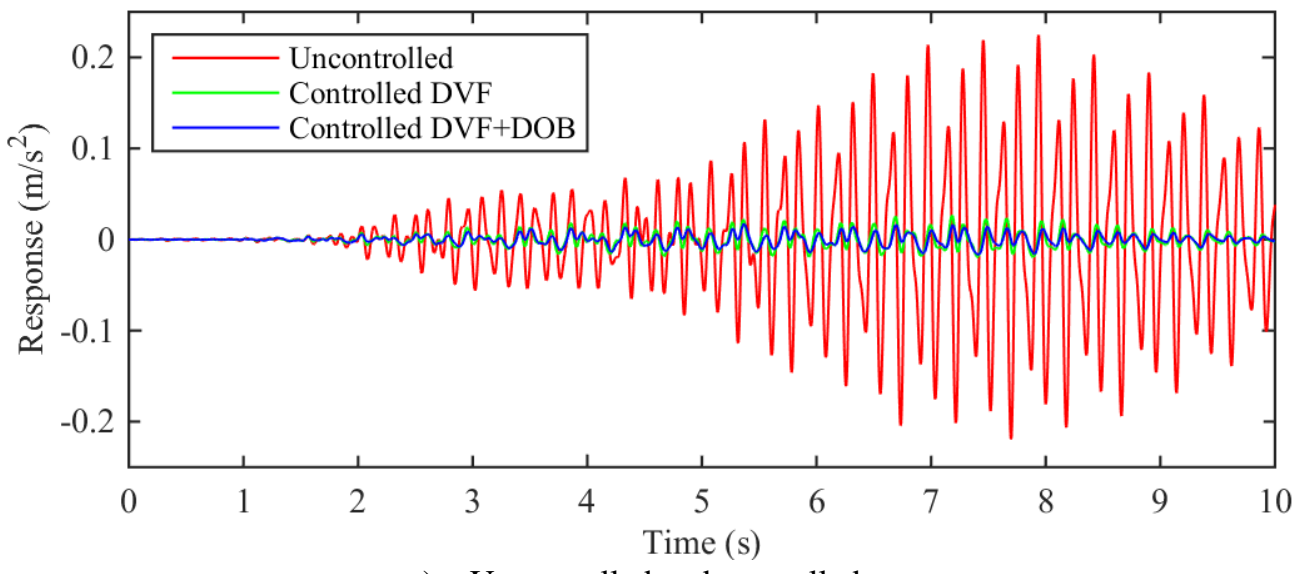

a) Uncontrolled and controlled responses

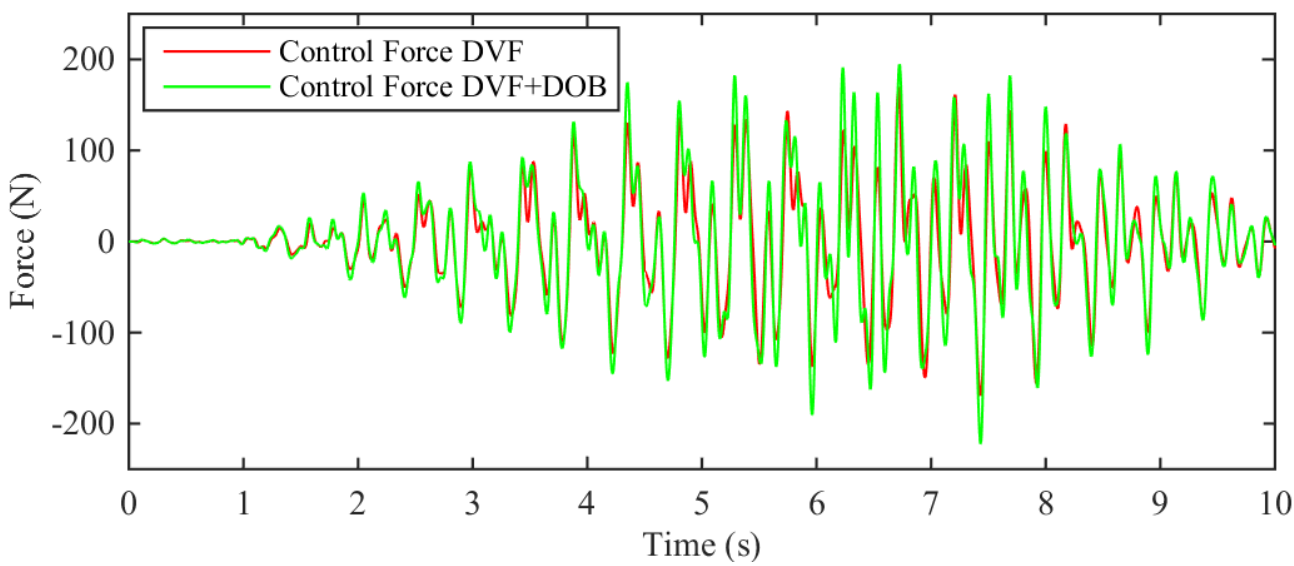

b) Actuator forces

Figure 9. Uncontrolled and controlled walkway bridge structural responses and actuator forces under synthesized walking excitation 1 


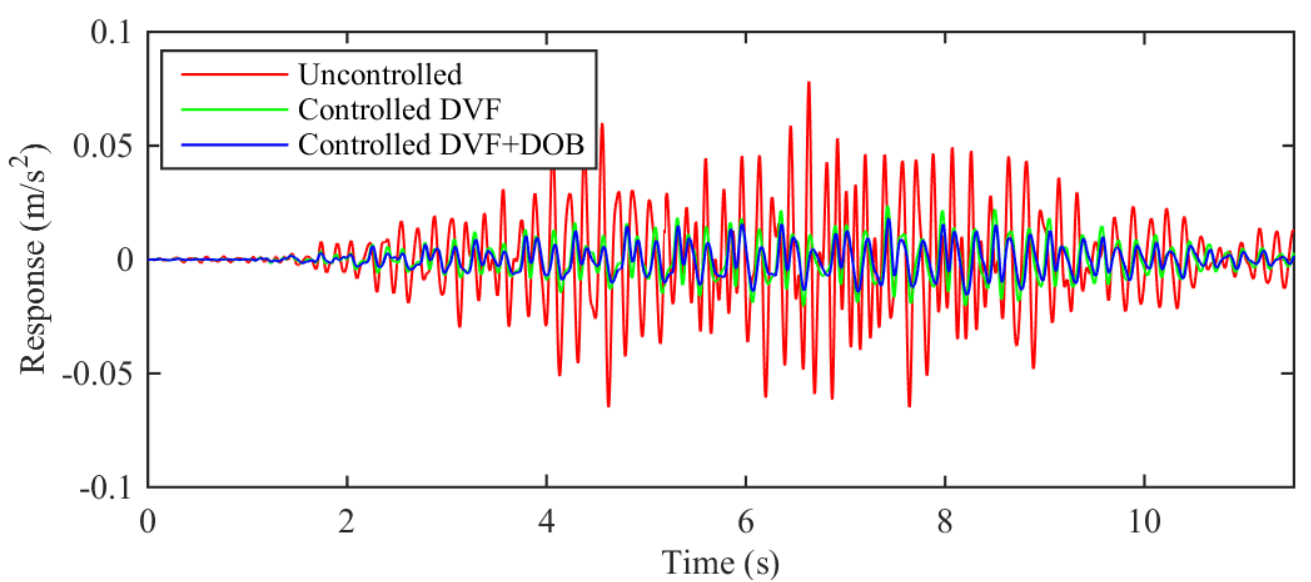

a) Uncontrolled and controlled responses

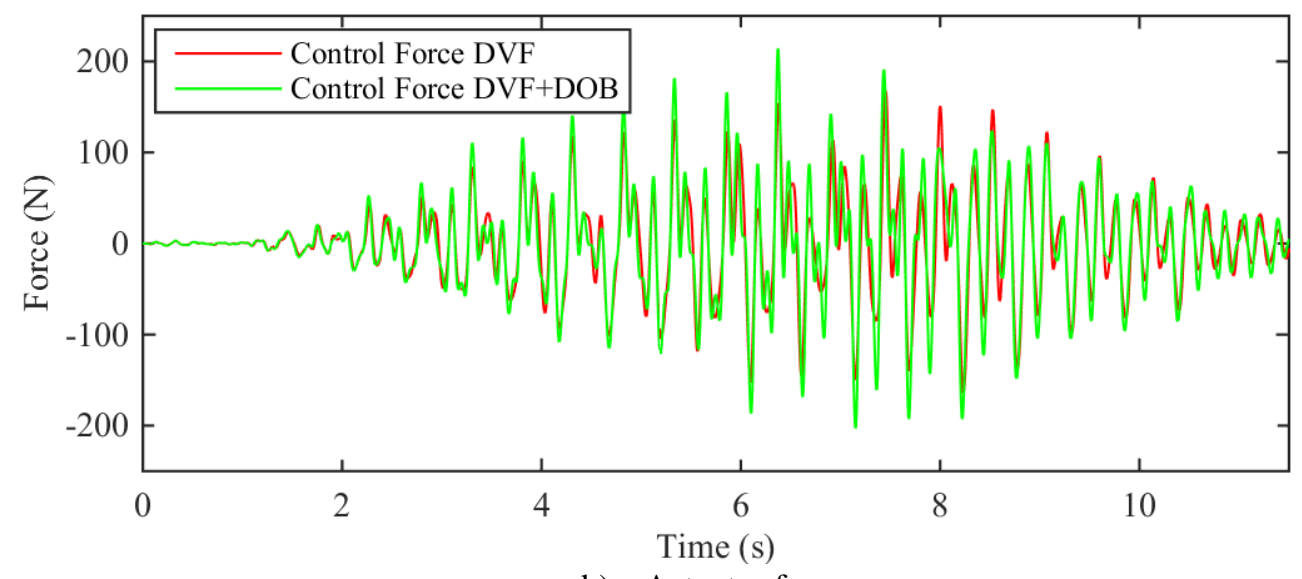

b) Actuator forces

Figure 10. Uncontrolled and controlled walkway bridge structural responses and actuator forces under synthesized walking excitation 2 


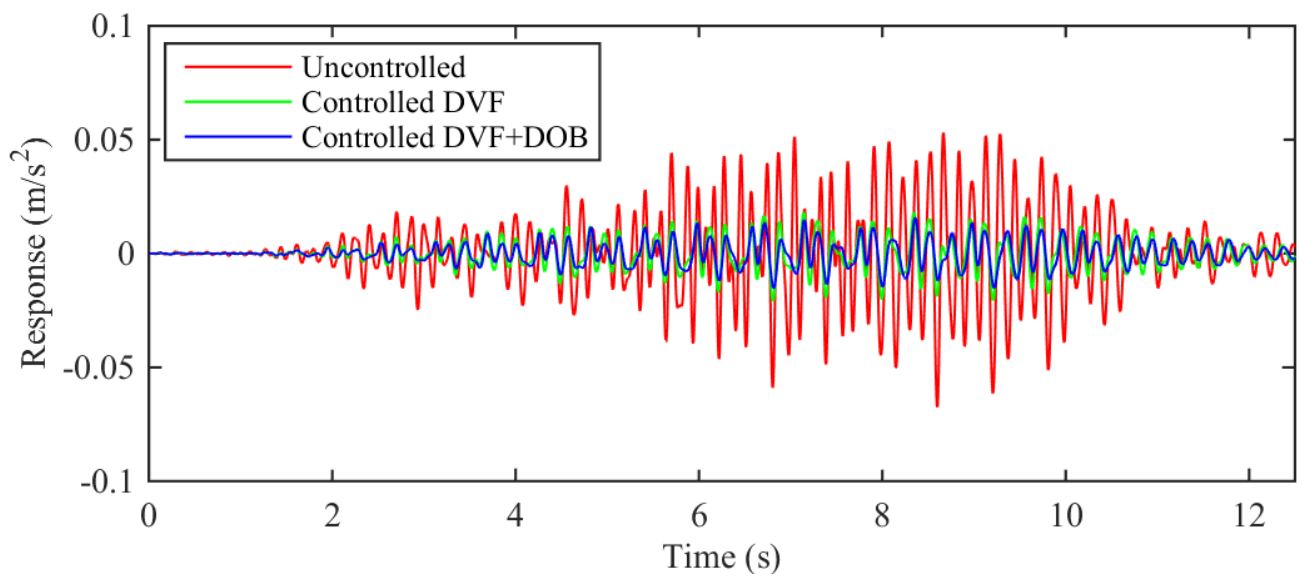

a) Uncontrolled and controlled responses

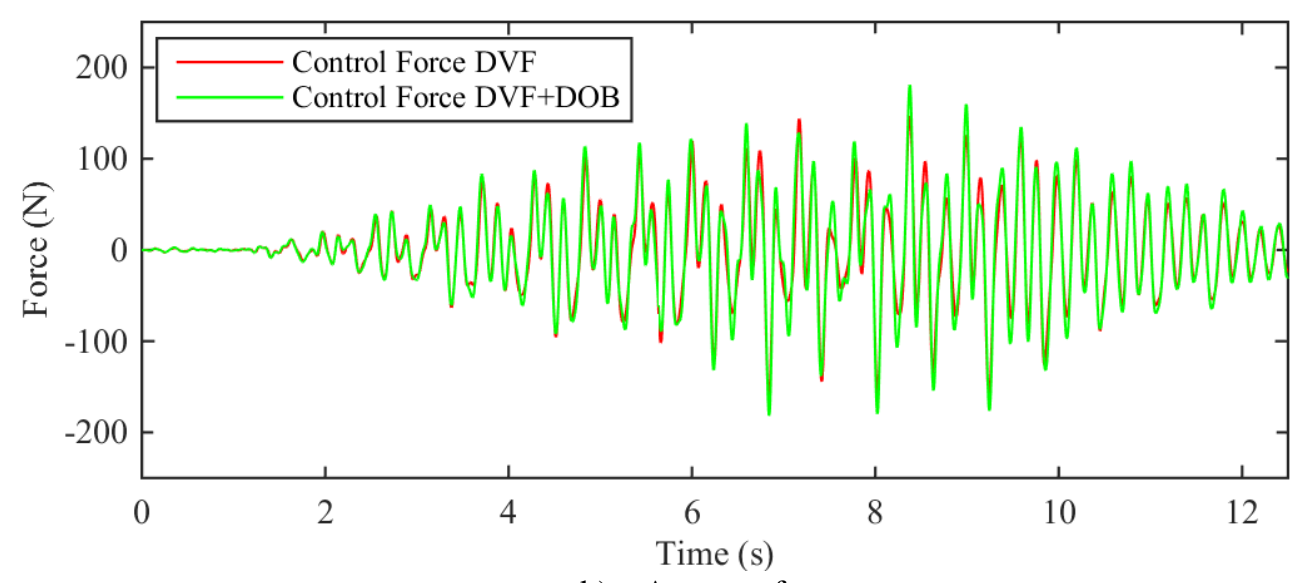

b) Actuator forces

Figure 11. Uncontrolled and controlled walkway bridge structural responses and actuator forces under synthesized walking excitation 3

Table 2 highlights potential vibration mitigation performances of DVF and DVF+DOB controllers implemented of an analytical model of a walkway bridge structure subjected to three synthesized walking excitation forces. It is seen that the DVF+DOB controller offers a general improvement in the vibration mitigation performance for all the three synthesized walking excitation forces considered and this ranges from about $3.5 \%$ to $10.0 \%$. Table 3 also reflects that appropriate compensation has been provided to deal with potential actuator stroke saturation instability.

Table 2. Peaks of the 1s running RMS acceleration responses simulated for uncontrolled and controlled structural models to walking excitation frequencies 1, 2 and 3

\begin{tabular}{|l|c|c|c|}
\hline & $\begin{array}{c}\text { Uncontrolled } \\
\text { response }\left(\mathrm{m} / \mathrm{s}^{2}\right)\end{array}$ & $\begin{array}{c}\text { Controlled response }\left(\mathrm{m} / \mathrm{s}^{2}\right) \\
\text { DVF } \\
\text { (reduction in parentheses) }\end{array}$ & $\begin{array}{c}\text { Controlled response }\left(\mathrm{m} / \mathrm{s}^{2}\right) \\
\text { DVF + DOB } \\
\text { (reduction in parentheses) }\end{array}$ \\
\hline Walking frequency 1 & 0.1345 & $0.0140(89.6 \%)$ & $0.0090(93.3 \%)$ \\
\hline Walking frequency 2 & 0.0327 & $0.0129(60.6 \%)$ & $0.0095(71.0 \%)$ \\
\hline Walking frequency 3 & 0.0383 & $0.0114(70.2 \%)$ & $0.0089(76.8 \%)$ \\
\hline
\end{tabular}

Table 3. Simulated actuator displacements under walking excitation forces 1, 2 and 3

\begin{tabular}{|c|c|c|}
\hline & $\begin{array}{c}\text { Actuator displacement } \\
(\mathrm{mm})-\mathrm{DVF}\end{array}$ & $\begin{array}{c}\text { Actuator displacement } \\
(\mathrm{mm})-\mathrm{DVF}+\mathrm{DOB}\end{array}$ \\
\hline Walking frequency 1 & 15.11 & 19.20 \\
\hline Walking frequency 2 & 18.92 & 21.14 \\
\hline Walking frequency 3 & 21.11 & 24.70 \\
\hline
\end{tabular}




\section{CONCLUSIONS}

The analytical studies presented in this work have looked at comparative studies between a purely direct velocity feedback (DVF) controller and a direct velocity feedback controller with an inner loop disturbance observer (DVF+DOB) for the control of vibrations on a walkway bridge structure. From the controlled frequency response function (FRF) estimates, there appears to be a great potential of adding a DOB to an outer loop DVF controller to deal with off-resonance excitation forces within a certain frequency bandwidth that may be introduced from the harmonics of walking excitation. Such improvements are greatly influenced by compensation provided for the actuator dynamics to deal with potential stroke saturation at the actuator resonant frequency. There also appears to be greater potential offered by a DVF+DOB in suppressing higher structural resonant frequencies beyond what is achievable with a purely DVF controller.

Studies of analytical simulations with three synthesized walking excitation forces at different pacing frequencies highlight the observations seen in the FRF estimates. They show the potential improvements in the vibration mitigation performance of a DVF+DOB controller over a purely DVF controller, with improvements in the range of $3.5-10.0 \%$ as reflected in Table 3. As seen in the present studies, a DOB can thus be incorporated with an outer loop DVF controller to augment its vibration mitigation performance over a broader range of frequencies. Hard constraints are introduced to deal with potential stroke saturation instability of the APS actuators.

\section{ACKNOWLEDGEMENTS}

The authors would like to acknowledge the financial assistance provided by the UK Engineering and Physical Sciences Research Council (EPSRC) through a responsive mode grant (Ref. EP/H009825/1) and a Leadership Fellowship Grant (Ref. EP/J004081/2).

\section{REFERENCES}

[1] Craig, K.C. Control design: pervasive and perplexing, Mechatronics in Design, EDN Design News (2010)

[2] Díaz, I. M., Pereira, E., Hudson, M. J., \& Reynolds, P. (2012). Enhancing active vibration control of pedestrian structures using inertial actuators with local feedback control. Engineering Structures, 41, 157-166. doi:10.1016/j.engstruct.2012.03.043

[3] Hanagan, L. M., \& Murray, T. M. (1997). Active Control Approach for Reducing Floor Vibrations. Journal of Structural Engineering, 123(11), 1497-1505. doi:10.1061/(ASCE)0733-9445(1997)123:11(1497)

[4] Hudson, M.J., \& Reynolds, P. (2013). Potential benefits of incorporating active vibration control in floor structures. The Structural Engineer, 91(2), 46-48.

[5] Bagordo, G., Cazzulani, G., Resta, F., \& Ripamonti, F. (2011). A modal disturbance estimator for vibration suppression in nonlinear flexible structures. Journal of Sound and Vibration, 330(25), 6061-6069. doi:10.1016/j.jsv.2011.07.014

[6] Wang, D.-A., \& Huang, Y.-M. (2002). Modal space vibration control of a beam by using the feedforward and feedback control loops. International Journal of Mechanical Sciences, 44(1), 1-19. doi:10.1016/S00207403(01)00088-1

[7] Yamada, K., Murakami, I., Ando, Y., Hagiwara, T., \& Imai, Y. (2009). The parametrization of all disturbance observers for plants with input disturbance. 2009 4th IEEE Conference on Industrial Electronics and Applications, ICIEA 2009, 41(1), 41-46. doi:10.1109/ICIEA.2009.5138167

[8] Schrijvert, E., Dijkt, J. Van, \& Nijmeijert, H. (2000). Equivalence of disturbance observer structures for linear systems. Proceedings of the 39th IEEE Conference on Decision and Control, Sydney, Australia, December 2000, 4518-4519. Retrieved from http://ieeexplore.iee.org/stamp/stamp.jsp?tp=\&arnumber=914622

[9] Profeta, J.A., Vogt, W.G. \& Mickle, M. H. (1990). Disturbance estimation and compensation in linear systems. IEEE Transactions on Aerospace and Electronic Systems, 26(2), 225-231.

[10] Coelingh, H. J., Schrijver, E., de Vries, T. J. A., \& van Dijk, J. (2000). Design of disturbance observers for the compensation of low-frequency disturbances. Proc. 5th Int. Conf. on Motion and Vibration Control (MOVIC 2000), 1, 75-80. 
[11] Kempf, C. J., \& Kobayashi, S. (1999). Disturbance observer and feedforward design for a high-speed directdrive positioning table. IEEE Transactions on Control Systems Technology, 7(5), 513-526. doi:10.1109/87.784416

[12] Morales, R., Feliu, V., \& Jaramillo, V. (2012). Position control of very lightweight single-link flexible arms with large payload variations by using disturbance observers. Robotics and Autonomous Systems, 60(4), 532-547. doi:10.1016/j.robot.2011.11.016

[13] Nyawako, D. S., \& Reynolds, P. (2015). Observer-based controller for floor vibration control with optimization algorithms. Journal of Vibration and Control, (August 2014). doi:10.1177/1077546315581229

[14] ISO 2631:1997 Mechanical vibration and shock evaluation of human exposure to whole body vibration. 